\title{
Robotic Assisted Laparoscopic Apical Suspension. Description of a 4 Points Technique (RALAS-4): First Case Reported
}

\author{
Hugo H Davila1,2,3, Lindsey Bruce², Lindsey Goodman², Taryn Gallo² \\ ${ }^{1}$ Florida Healthcare Specialist, Urology and Minimally Invasive Surgery, Florida Cancer Specialist \& Research Institute, Sebastian \\ and Vero Beach, USA \\ ${ }^{2}$ Department of Surgery, Division of Urology and Gynecology, Sebastian River Medical Center, Sebastian, USA \\ ${ }^{3}$ Florida State University College of Medicine, Fort Pierce Campus, Fort Pierce, USA \\ Email: Hdavila@FLHealthcarespecialists.com
}

How to cite this paper: Davila, H.H. Bruce, L., Goodman, L. and Gallo, T. (2017) Robotic Assisted Laparoscopic Apical Suspension. Description of a 4 Points Technique (RALAS-4): First Case Reported. Open Journal of Obstetrics and Gynecology, 7, 944-950.

https://doi.org/10.4236/ojog.2017.79095

Received: August 9, 2017

Accepted: September 9, 2017

Published: September 12, 2017

Copyright (c) 2017 by authors and Scientific Research Publishing Inc. This work is licensed under the Creative Commons Attribution International License (CC BY 4.0).

http://creativecommons.org/licenses/by/4.0/

\begin{abstract}
The uterosacral ligaments (UTSL), together with the cardinal ligament (CL), hold the upper vagina and cervix over the levator plate. These 2 ligaments provided 4 points support at the apex. Here we describe our surgical technique of robotic assisted laparoscopic apical suspension (RALAS) using nonabsorbable sutures and describe a new 4 points technique (RALAS-4). 73year-old Caucasian woman, gravida 5, para 4 had symptomatic pelvic organ prolapse (POP) apical/anterior stage III. At pelvic ultrasound evaluation the uterus was small and normal appearing of adnexa bilaterally. She failed pessaries and was sexually active. The most relevant complaints were vaginal bulging, pressure and urinary incontinence, mainly stress urinary incontinence; she is using $5-7$ pads/day. Robotic assisted laparoscopic hysterectomy, mid-urthral sling and apical suspension was successfully performed in 125 min. Once we finished with hysterectomy, we proceed with RALAS-4, we used V-Loc 3-0, CV-23 (Covidien) sutures (absorbable) on the right and left uterosacral ligaments (2 points) and theses were reinforced with Gore-Tex 2-0, CV-2 (non-absorbable, Gore Medical). On the right/left anterior apical support we used Gore-Tex 2-0 and these provided the 2 point suspension (UTLS $=2$ and anterior vagina $=2$ ). The 2 anterior apical support sutures are taken from the vagina to the transversalis fascia and the obliterated umbilical artery on the anterior abdominal wall. The tension of these anterior sutures was maintained with Hem-o-lock (TeleFlex) and LAPRA-TY (Ethicon). In our opinion RALAS-4 may represents an alternative to robotic or laparoscopic sacrocolpopexy. This new approach simulate the natural 4 points support given by uterosacral ligaments and cardinal ligament, with the additional benefit of
\end{abstract}


no mesh and no dissection on the sacrum promontory. With this technique we are chasing the Trifecta: no mesh, no complications and good anatomic support.

\section{Keywords}

Apical Prolapse, Vaginal Prolapse, Pelvic Anatomy, Robotic Surgery, Uterosacral Ligament, Cardinal Ligament

\section{Introduction}

The correction of pelvic organ prolapses (POP) has changed drastically during the last years. In 2008, the large number of reported adverse events with the transvaginal placement of mesh to correct POP prompted the FDA to issue a Public Health Notification outlining the potential serious consequences of such placement [1]. Due to concerns regarding the safety of vaginal meshes, there was a 7\% decrease in mesh use during vaginal surgery for POP in the 2 years following the 2011 FDA safety communication [2]. Laparoscopic sacrocolpopexy has been adopted by many pelvic surgeons as a way to minimize surgical morbidity and quicken patient recovery [3] [4] [5]. Abdominal sacrocolpopexy (ASC) has been shown to have one of the highest long-term anatomic success rates $(78 \%$ $100 \%$ ) among procedures for pelvic organ prolapse repair [6] with minimal complications [7]. During ASC identification of the pre-sacral ligament can be difficult, particularly in obese patients. This area is surrounded by critical structures, such as the right ureter, where injuries happen during ASC in $1.0 \%$ of the procedures $(0.8 \%-1.9 \%)$ [8]. The middle sacral vessels, the left iliac vein and the caval bifurcation are also nearby the area where the mesh needs to be placed. Bleeding management can be particularly difficult in this area and accidental lesions of these vessels can result in blood loss, which is described in $4.4 \%$ of the procedures $(0.18 \%-16.9 \%)$ [8]. Awareness of these challenges may discourage the use of ASC, therefore, limiting the access of women with advanced apical prolapse to the most effective surgical strategy available.

An additional concern about ASC, open or minimally invasive, is that has been associated with mesh erosion $2 \%$ - 7\% [7] [8]. Development of new surgical techniques that provide good long term support, minimal complications and avoid mesh utilization (Trifecta) is key to permit broader dissemination of minimally invasive abdominal procedures for advanced apical POP. We recently described our technique using non-absorbable sutures and avoiding mesh enhanced surgery for the correction of apical POP. We performed a series of cases with robotic and laparoendoscopic single-site utero-sacral ligament suspension and $92 \%$ of patients had good anatomic support at 12 months after surgery [9].

POP is caused by structural defects in the connective tissue and the muscles that support the pelvic viscera. The uterosacral ligaments have long been regarded as a part of this support system for the pelvic organs [10]. Nichols, in his 
book on vaginal surgery, expressed the widely held opinion that the uterosacral ligaments, together with the cardinal ligament, hold the upper vagina and cervix over the levator plate [11]. These 2 ligaments provided 4 points support at the apex. With the aim to continue exploring additional techniques without mesh and provide the best apical support, here we describe our surgical technique of robotic assisted laparoscopic apical suspension (RALAS) using non-absorbable sutures and describe a new 4 points technique (RALAS-4).

\section{Patient Characteristics}

In March 2017, a postmenopausal 73-year-old Caucasian woman (body mass index $30.7 \mathrm{~kg} / \mathrm{m}^{2}$ ), gravida 5 , para 4 had symptomatic apical/anterior POP stage III. Her medical history was significant for hypertension and hyperlipidemia, physical examination was within the normal limits, and complete blood count and basic metabolic profile were normal. At pelvic ultrasound evaluation the uterus was small and normal appearing of adnexa bilaterally. Patient reported no previous surgeries. She failed pessaries and is sexually active. The most relevant complaints were vaginal bulging, pressure and urinary incontinence, mainly stress urinary incontinence; she is using $5-7$ pads/day. We did a cystoscopy and CT-urogram due to microscopic hematuria, no evidence of cancer. We used the da Vinci Si systems (Intuitive Surgical, Sunnyvale, CA, USA) to perform the procedure and at the same time we placed mid urethral sling, Trans-obturator Tape (Obtryx II, Boston Scientific). Patient was counseled about the risks, benefits, and alternative treatments and signed an informed consent.

\section{Surgical Technique and Results}

At induction of anesthesia, the patient is placed in dorsal lithotomy position and bladder Foley catheter is inserted. Once the trocars were inserted, using 4 trocar, "W" configuration a $15 \mathrm{mmHg}$ pneumoperitoneum was established. After introducing the Da Vinci 30 optic (0 degree) and performing diagnostic laparoscopy, the patient is positioned in Trendelenburg position and the Da Vinci robot is laterally docked; hence we have easy access to the vagina during surgery. V-Care uterine manipulator (ConMed) was used during robotic assisted hysterectomy, and this was done following standard surgical technique (Figure 1). Robotic Assisted Laparoscopic hysterectomy and apical suspension was successfully performed in $125 \mathrm{~min}$. Once we finished with hysterectomy, we proceed with RALAS-4. Robotic assisted laparoscopic UTSL suspension procedure was performed following our technique recently published [9]. In brief, we used V-Loc 3-0, CV-23 (Covidien) sutures (absorbable) on the right and left UTSL and theses were reinforced with Gore-Tex 2-0, CV-2 (non-absorbable, Gore Medical), these are the first 2 points suspension. This was followed by the 2 anterior vaginal support with Gore-Tex, Hem-o-lock (TeleFlex) and LAPRA-TY (Ethicon) for a total 4 points apical support (Figure 2). The 2 apical support sutures are taken from the vagina to the transversalis fascia and the level of the ob- 
(a)

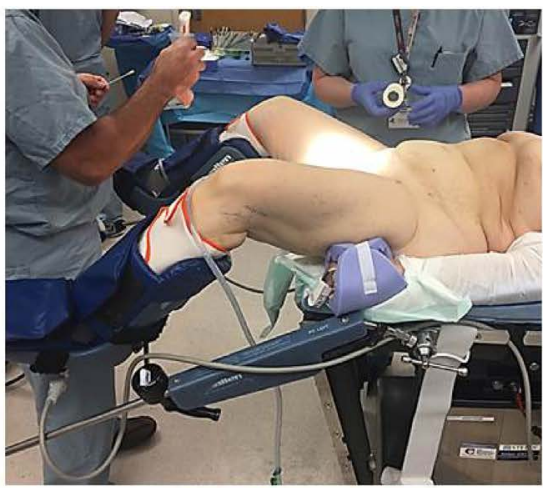

(d)

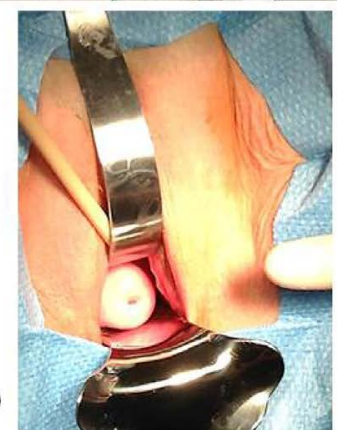

(b)

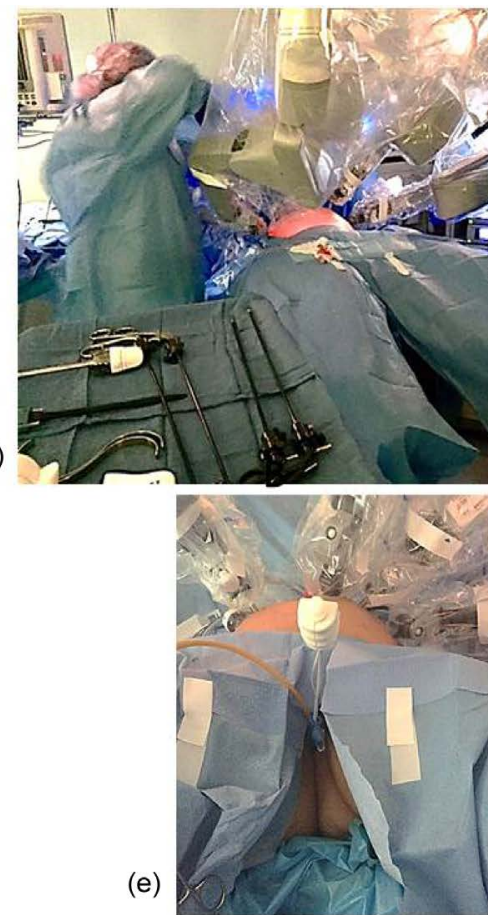

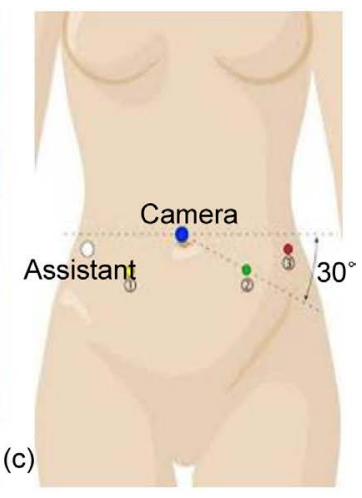

(c)

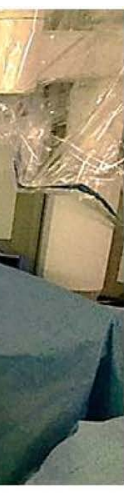

Figure 1. Patient images during robotic assisted laparoscopic apical suspension (RALAS). (a): Patient position, dorsal lithotomy; (b): The Da Vinci SI robot is laterally docked; (c): Trocars configuration; (d): Apical prolapse stage III; (e): V-Care uterine manipulator (ConMed) during surgery.

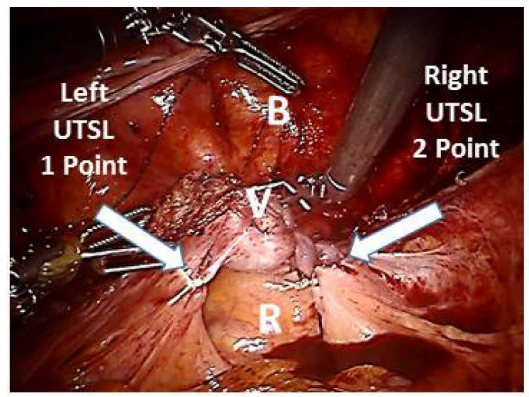

(a)

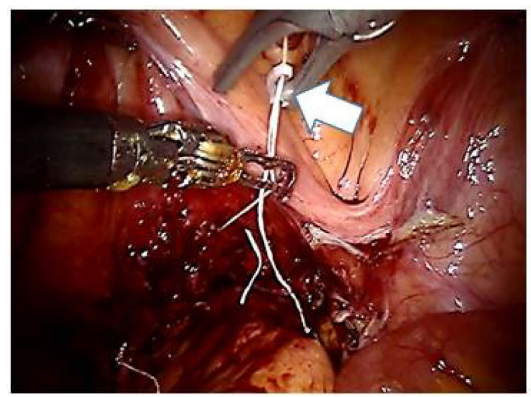

(d)

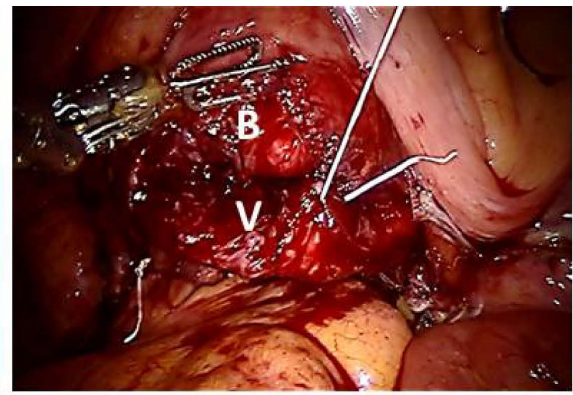

(b)

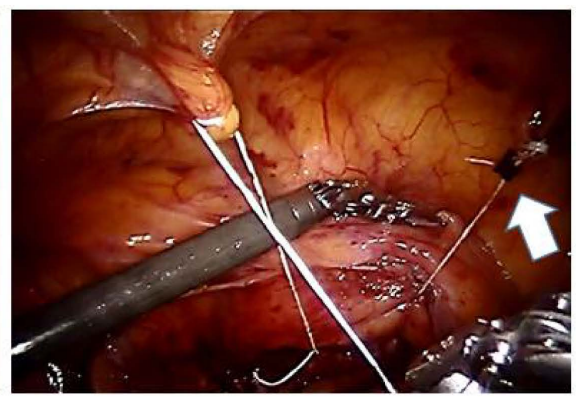

(e)

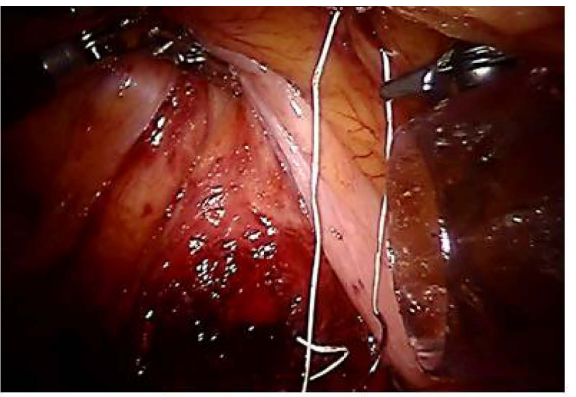

(c)

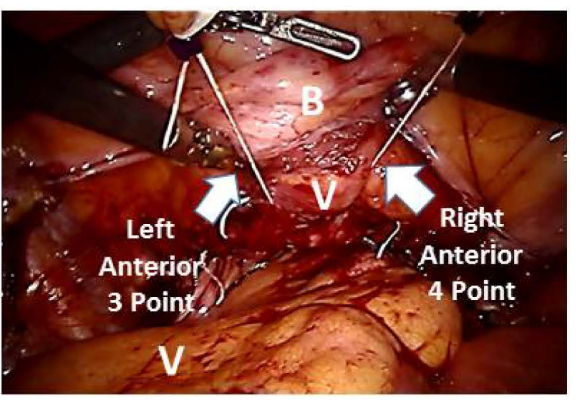

(f)

Figure 2. Images during robotic assisted laparoscopic apical suspension, 4 points (RALAS-4) after hysterectomy. (a): Right and left UTSL suspension with V-loc and Gore-Tex (2 point suspension) white arrows; (b): Demonstrates the right anterior vagina suture (Gore-Tex); (c): Suture from vagina to anterior abdominal wall; (d): To keep the tension we uses Hem-o-lock (TeleFlex), white arrow; (e): We uses LAPRA-TY (Ethicon) white arrow, to hold the Hem-o-lock; (f): Right and left anterior vaginal suspension (2 point suspension), white arrows, the location of the bladder (B) and vagina (V) are shown. 
literated umbilical artery on the anterior abdominal wall (right/left) (Figure 3). The postoperative course was unremarkable. Catheter was removed next morning after surgery. The patient reported minimal postoperative pain and was discharged from the hospital $24 \mathrm{~h}$ after surgery. At discharge, patient had an appointment scheduled at 2 and 4 weeks and then at three months. No POP recurrence at 3 months and she is $100 \%$ dry.

\section{Discussion}

Robotic assisted laparoscopic apical suspension, 4 points technique (RALAS-4) without mesh is an innovative technique with promising results for the treatment of advanced anterior and apical prolapse. Our previous experience using 2 points approach shows that the procedure is feasible and safe and mirrors our previous experience on a series of cases performed with robotic and laparoscopic single site UTSL suspension platform [9].

The advantages of this technique are the following: a) provide 4 apical support, b) avoid mesh and c) potentially decreases complications associated with ASC is a substantial evolution (Trifecta). This approach offer the benefits of minimally invasive surgery: including increased dexterity, range of motion, instrument and scope stability, ergonomics, 3D visualization, surgical field magnification and easy dissection and suturing. All these technical improvements may shorten the learning curve and might represent the basis for wider utilization of this surgical technique.

The uterosacral ligaments have long been regarded as a part of the support system for the pelvic organs [10]. Nichols, in his book on vaginal surgery, expressed the widely held opinion that the uterosacral ligaments, together with the cardinal ligament, hold the upper vagina and cervix over the levator plate [11]. Anatomically, apical support is provided by the combined action of the cardinal

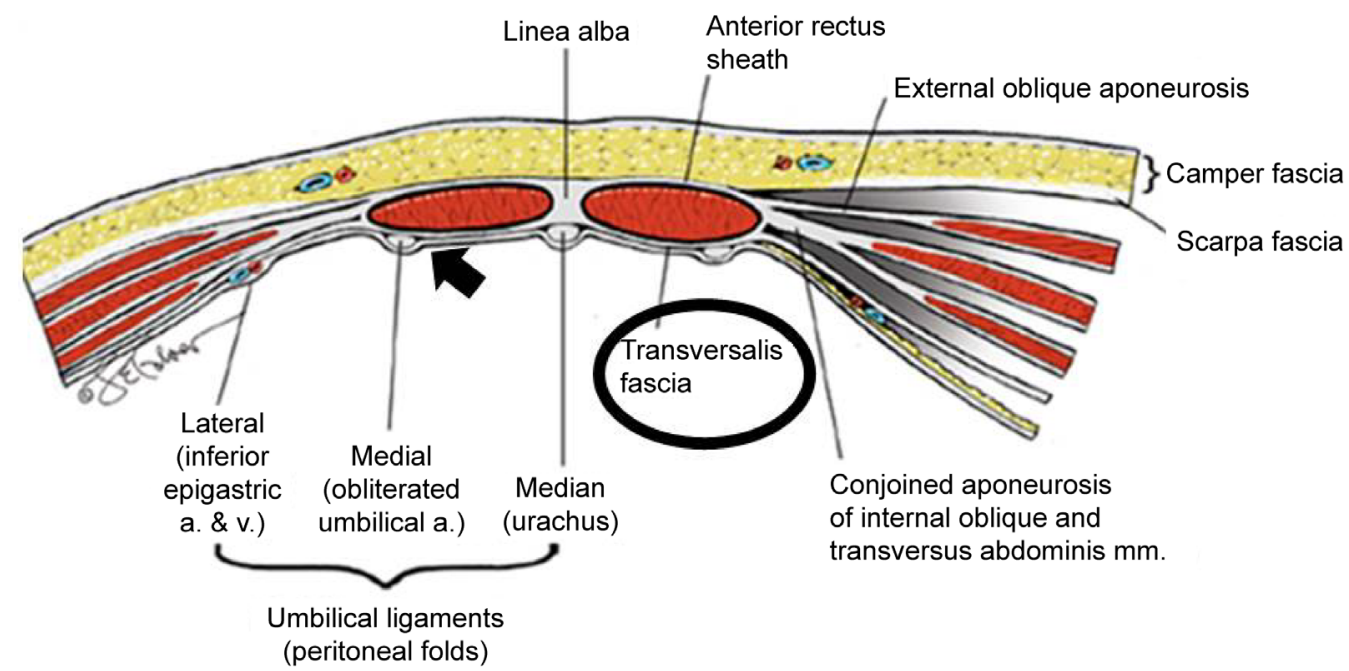

Figure 3. Abdominal wall anatomy and location of the Transversalis Fascia. The 2 apical support sutures are taken from the vagina to the transversalis fascia (black circle) and the obliterated umbilical artery (black arrow) on the anterior abdominal wall. 
and uterosacral ligaments [12]. The structure of these ligaments has been described based on cadaveric dissections and cross-sectional anatomy [13]. Therefore, we are proposing this 4 points technique; to try to simulate the similar support given by the UTSL and CL (right and left side). Understanding that one of the main limitation are the external iliac vessels that prevent us to provide a lateral support with sutures similar to the natural CL insertion (CL insert on the lateral wall); hence we are using the transversalis fascia and the obliterated umbilical artery to anchor the apical sutures, together with UTSL ligament suspension as previously described [9] and this provide the 4 points support. There are some arguments to consider in this study. Firstly, surgery was performed on the dorsal lithotomy position; this may not accurately reflect the pelvic floor anatomy in the upright position. Second, we did the 2 apical suspension with a pneumoperitoneum pressure of $15 \mathrm{mmHg}$, to avoid over-tension of the sutures and allow some sutures movement when patients are on upright position.

\section{Conclusion}

POP is a common condition with an estimated incidence of up to $40 \%$ of women and shows a growing prevalence in Western countries due to increased life expectancy [14]. 30\% of women aged 50 - 89 years require a consultation for pelvic floor dysfunction and the life-time risk of surgical repair is estimated at $11 \%$, with almost one-third of the patients requiring repeat surgery. In our opinion RALAS-4 may represents an alternative to robotic or laparoscopic ASC. This new approach simulates the natural 4 points support given by UTSL and CL, with the additional benefit of no mesh and no dissection on the sacrum promontory close to the iliac vessels and right ureter. With this technique we are chasing the Trifecta: no mesh, no complications and good anatomic support. We are collecting data to continue the evaluation of the long-term operative outcomes of this technique and compare it with our robotic sacrocolpopexy.

\section{Conflicts of Interest}

Authors have nothing to disclose.

\section{Compliance with Ethical Standards and Informed Consent}

Informed consent was obtained from all individuals included in the study.

\section{References}

[1] Division of Small Manufacturers, International and Consumer Assistance (DSMICA) (2011) Update on Serious Complications Associated with Transvaginal Placement of Surgical Mesh for Pelvic Organ Prolapse: FDA Safety Communication. Silver Spring (MD, USA). US Food and Drug Administration.

https://www.fda.gov/newsevents/newsroom/pressannouncements/ucm479732.htm

[2] Sedrakyan, A., Chughtai, B. and Mao, J. (2016) Regulatory Warnings and Use of Surgical Mesh in Pelvic Organ Prolapse. JAMA Internal Medicine, 176, 275-277. http://jamanetwork.com/journals/jamainternalmedicine/fullarticle/2475021 
https://doi.org/10.1001/jamainternmed.2015.6595

[3] Cosson, M., Rajabally, R., Bogaert, E., Querleu, D. and Crepin, G. (2002) Laparoscopic Sacrocolpopexy, Hysterectomy, and Burch Colposuspension: Feasibility and Short-Term Complications of 77 Procedures. Journal of the Society of Laparoendoscopic Surgeons, 6, 115-119

[4] Nezhat, C.H., Nezhat, F. and Nezhat, C. (1994) Laparoscopic Sacral Colpopexy for Vaginal Vault Prolapse. Obstetrics \& Gynecology, 84, 885-888.

[5] Ostrzenski, A. (1996) Laparoscopic Colposuspension for Total Vaginal Prolapse. International Journal of Gynecology \& Obstetrics, 55, 147-152. https://doi.org/10.1016/S0020-7292(96)02731-2

[6] Nygaard, I.E., McCreery, R., Brubaker, L., Connolly, A.M., Cundiff, G., Weber, A.M., et al. (2004) Abdominal Sacrocolpopexy: A Comprehensive Review. Obstetrics \& Gynecology, 104, 805-823. https://doi.org/10.1097/01.AOG.0000139514.90897.07

[7] Serati, M., Bogani, G., Sorice, P., Braga, A., Torella, M., Salvatore, S., Uccella, S., Cromi, A. and Ghezzi, F. (2014) Robot-Assisted Sacrocolpopexy for Pelvic Organ Prolapse: A Systematic Review and Meta-Analysis of Comparative Studies. European Urology, 66, 303-318. https://doi.org/10.1016/j.eururo.2014.02.053

[8] Nygaard, I.E., McCreery, R., Brubaker, L., et al. (2004) Pelvic Floor Disorders Network. Abdominal Sacrocolpopexy: A Comprehensive Review. Obstetrics \& Gynecology, 104, 805-823. https://doi.org/10.1097/01.AOG.0000139514.90897.07

[9] Davila, H.H., Gallo, T., Bruce, L. and Landrey, C. (2016) Robotic and Laparoendoscopic Single-Site Utero-Sacral Ligament Suspension for Apical Vaginal Prolapse: Evaluation of Our Technique and Perioperative Outcomes. Journal of Robotic Surgery, 11, 171-177.

[10] Singh, K., Jakab, M., Reid, W.M., Berger, L.A. and Hoyte, L. (2003) Three-Dimensional Magnetic Resonance Imaging Assessment of Levator Ani Morphologic Features In Different Grades of Prolapse. American Journal of Obstetrics \& Gynecology, 188, 910-915. https://doi.org/10.1067/mob.2003.254

[11] Nichols, D.H. and Randall, C.L. (1996) Vaginal Surgery. 4th Edition, Williams \& Wilkins, Baltimore, 101-118.

[12] DeLancey, J.O. (1992) Anatomic Aspects of Vaginal Eversion after Hysterectomy. American Journal of Obstetrics \& Gynecology, 166, 1717-1784. https://doi.org/10.1016/0002-9378(92)91562-O

[13] Blaisdell, F.E. (1917) The Anatomy of the Sacro-Uterine Ligaments. The Anatomical Record, 12, 1-42. https://doi.org/10.1002/ar.1090120102

[14] Gerten, K.A., Markland, A.D., Lloyd, L.K., et al. (2008) Prolapse and Incontinence Surgery in Older Women. The Journal of Urology, 179, 2111-2121.

https://doi.org/10.1016/j.juro.2008.01.089 
Submit or recommend next manuscript to SCIRP and we will provide best service for you:

Accepting pre-submission inquiries through Email, Facebook, LinkedIn, Twitter, etc. A wide selection of journals (inclusive of 9 subjects, more than 200 journals)

Providing 24-hour high-quality service

User-friendly online submission system

Fair and swift peer-review system

Efficient typesetting and proofreading procedure

Display of the result of downloads and visits, as well as the number of cited articles Maximum dissemination of your research work

Submit your manuscript at: http://papersubmission.scirp.org/

Or contact ojog@scirp.org 